\title{
MAGNETIC RESONANCE IMAGING FEATURES OF INTRACRANIAL ASTROCYTOMAS AND OLIGODENDROGLIOMAS IN DOGS
}

Benjamin D. Young ${ }^{1}$, Jonathan M. Levine ${ }^{1}$, Brian F. Porter ${ }^{1}$, Annie V. Chen-Allen ${ }^{2}$, John H. Rossmeisl ${ }^{3}$, Simon R. Platt ${ }^{4}$, Marc Kent ${ }^{4}$, Geoffrey T. Fosgate ${ }^{5}$, Scott J. Schatzberg ${ }^{4}$. ${ }^{1}$ Texas A\&M University, College Station, TX, ${ }^{2}$ Washington State University, Pullman, WA, ${ }^{3}$ Virginia Tech, Blacksburg, VA, ${ }^{4}$ University of Georgia, Athens, GA, and ${ }^{5}$ University of Pretoria, Onderstepoort, South Africa.

Running head: MRI of Astrocytomas and Oligodendrogliomas

Abstract will be presented at the 2010 Annual Scientific Convention of the ACVR in Asheville, NC.

\begin{abstract}
Astrocytomas and oligodendrogliomas represent one third of histologically confirmed canine brain tumors. Our purpose was to describe the magnetic resonance (MR) imaging features of histologically confirmed canine intracranial astrocytomas and oligodendrogliomas and to examine for MR features that differentiate these tumor types. Thirty animals with confirmed astrocytoma (14) or oligodendroglioma (16) were studied. All oligodendrogliomas and 12 astrocytomas were located in the cerebrum or thalamus, with the remainder of astrocytomas in the cerebellum or caudal brainstem. Most (27/30) tumors were associated with both grey and white matter. The signal characteristics of both tumor types were hypointense on T1-W (12 each) and hyperintense on T2-W
\end{abstract}


(11/14 astrocytomas, 12/16 oligodendrogliomas). For astrocytomas and oligodendrogliomas, respectively, common findings were contrast enhancement (10/13, 11/15), ring-like contrast enhancement $(6 / 10,9 / 11)$, cystic regions within the mass $(7 / 14,12 / 16)$, and hemorrhage $(4 / 14,6 / 16)$. Oligodendrogliomas were significantly more likely to contact the brain surface (meninges) than astrocytomas $(14 / 16,7 / 14$, respectively, $P=0.046)$. Contact with the lateral ventricle was the most common finding, occurring in 13/14 astrocytomas and 14/16 oligodendrogliomas. No MR features were identified that reliably distinguished between these two tumor types. Contrast enhancement was more common in high grade tumors (III or IV) than low grade tumors (II, $P=0.008)$.

\section{Introduction}

Astrocytomas and oligodendrogliomas are glial tumors and account for $31 \%$ of primary brain tumors in dogs. ${ }^{1}$ The World Health Organization (WHO) recognizes low and high grade variants of astrocytoma (e.g., diffuse astrocytoma (II), anaplastic astrocytoma (III), glioblastoma multiforme (IV)) and oligodendrogliomas (e.g., oligodendroglioma (II), anaplastic oligodendroglioma (III)). ${ }^{2}$ Despite the common occurrence of these tumors in dogs and humans, treatment strategies are rarely curative and survival is poor. ${ }^{1,3}$

MR imaging features may sometimes be a non-invasive surrogate for determining tumor type, especially when biopsy is not feasible. MR imaging is highly specific in differentiating human oligodendroglioma from astrocytoma and often provides information regarding tumor grade. ${ }^{4}$ Differentiation between tumor type and grade is important, as survival differs significantly among these groups. ${ }^{5}$ Mean survival for 
human patients with low-grade (II) oligodendroglioma is twice that of patients with lowgrade (II) astrocytoma, and survival for high-grade oligodendrogliomas (III) is over 8 times longer than those with high-grade astrocytoma (IV). ${ }^{6}$ Currently these survival data are largely unknown in dogs with astrocytomas or oligodendrogliomas. As attempts are made to determine survival and response of gliomas to current treatments, ${ }^{3,7-8}$ the need for establishing reliable MR imaging criteria to diagnose and distinguish between tumor types is likely to increase.

The MR criteria for diagnosing human astrocytomas and oligodendrogliomas include location, tissue involvement relative to grey matter, signal intensity and contrast enhancement. ${ }^{9}$ Oligodendrogliomas are heterogeneous in signal, typically isointense to grey matter, and are peripheral intra-axial masses. ${ }^{9}$ Cortical infiltration and marked cortical thickening is the most useful MR finding relating to the diagnosis of oligodendroglioma. ${ }^{9}$ In contrast, astrocytomas arise within white matter and are therefore located more deeply within the hemisphere, are typically T2-hyperintense to grey matter and are usually more homogeneous in signal intensity. Both glioma types may have internal hemorrhage, mineralization, and cystic areas, though cysts are less common in lower grade astrocytomas. ${ }^{9}$ Contrast enhancement is common to both and is usually non-uniform. High grade varieties of each tumor type (glioblastoma and anaplastic oligodendroglioma) can be indistinguishable on $\mathrm{MR}$, being highly heterogeneous and having extensive surrounding edema. ${ }^{9}$

In dogs, several reports and review articles exist concerning the MR appearance of intracranial glioma. ${ }^{1,10-14}$ Limited attempts have been made to differentiate between glioma types. The majority of intracranial gliomas reported in dogs are intra-axial, T2- 
hyperintense, solitary, mass-like lesions with variable T1 signal and post-contrast enhancement. ${ }^{1,10-14}$ Methodologic evaluation schemes for tumor diagnosis have been detailed previously,10,12-15 and partially mirror the approach used in people. ${ }^{9}$ The limitations of adopting these MR criteria for the dog include the small sample size of existing descriptions ${ }^{10}$, heterogeneous reporting of imaging findings between series, and grouping of tumor type data in some reports ${ }^{12-13,15}$. Little information has been included as to the peripheral location of oligodendrogliomas or the presence or absence of cortical thickening.

Our goals were to describe the MR imaging features of histologically confirmed canine intracranial astrocytomas and oligodendrogliomas through a multi-center series and to attempt to differentiate between glial tumor types based on imaging features. We hypothesized that: 1) oligodendrogliomas would be located more peripherally and more commonly contact the meninges, 2) astrocytomas would be located more deeply within the hemispheres and more commonly contact the lateral ventricles or thalamus, 3) signal intensity, the presence of hemorrhage, cystic areas, and contrast enhancement would be similar in both tumor types, 4) perilesional edema would be more common in high grade gliomas, and that 5) lesion burden would not be different between tumor types.

\section{Materials and Methods}

This retrospective study was an inter-institutional collaboration. The medical records of four veterinary teaching hospitals were searched for years 1996-2009. Dogs were included if they had: 1) antemortem brain MR imaging, and 2) histologic diagnosis of 
oligodendroglioma or astrocytoma. Thirty- dogs with a glial were identified. There were 14 astrocytomas and 16 oligodendrogliomas. Among dogs with astrocytoma, seven were neutered males and seven were neutered females. Dogs with oligodendrogliomas included four neutered males, eleven neutered females, and one intact female. Breeds were Boxer (9), Boston Terrier (5), mixed (3), English Bulldog (2), Golden Retriever (2), Labrador Retriever (2), American Bulldog (1), Australian Shepherd (1), Chesapeake Bay Retriever (1), French Bulldog (1), German Shepherd Dog (1), American Staffordshire Terrier (1), and Welsh Corgi (1).

A standard database for each animal was collected, including: signalment, time from onset of clinical signs to MR (days), results of cerebrospinal fluid (CSF) analysis, survival to discharge (yes/no), and survival time (days). The onset of clinical signs was defined as the first recognizable neurological signs referable to intracranial dysfunction. Survival time was the period from the onset of clinical signs to death.

MR images were acquired on high and low field systems, including: 0.2T, 1.0T, $1.5 \mathrm{~T}$, and $3.0 \mathrm{~T}$. With the exception of $3 \mathrm{~T}$ studies, all others included at least precontrast spin echo T1-weighted images (T1-W; TR 330-1040ms; TE 14-27ms) and spin echo or turbo spin echo T2-weighted images (T2-W; TR 2500-5633ms; TE 80-120ms) in transverse planes. Post-contrast T1-W were acquired in 28 of the 30 dogs. At $3 \mathrm{~T} \mathrm{T1}$ fluid attenuated inversion recovery (T1-FLAIR; TR 2584-2612ms; TE 8.3-8.4ms) sequences were substituted for pre- and post-contrast spin echo T1-W. Most studies also included sagittal and dorsal plane images, though not always in all sequences. Matrix size ranged from $192-512 \times 192-512$, but were most commonly $256 \times 256$. The 
number of excitations (NEX), brand, and dose of contrast medium were generally not reported. In 16 dogs, T2* gradient recall echo (GRE) images were acquired.

All images were evaluated in digital format by two investigators, a board certified radiologist (BDY) and a board certified neurologist (JML). Each evaluator reviewed the images independently and recorded MR findings according to a pre-established list of criteria. Evaluators were aware that lesions were gliomas but were unaware of type or grade. Interpretations were compared. Twenty-eight features were evaluated for each of thirty dogs (840 total scores). In 11 instances of initial discrepancy between reviewers, final designation was reached by consensus agreement. Lesions were defined as disruption of normal anatomy or change in tissue signal characteristics. Lesion number and location were recorded according to brain region, defined as the prosencephalon (telencephalon and diencephalon), cerebellum, or caudal brainstem (mesencephalon, ventral metencephalon (pons), and myelencephalon). Specific cerebral lobe (as assessed according to previously suggested landmarks ${ }^{16}$ ) and grey or white matter involvement were recorded. Lesion signal characteristics compared to normal grey matter were recorded.

The presence of contrast enhancement was recorded and graded subjectively as mild, moderate, or severe. ${ }^{10}$ Evidence of ring-like enhancement and perilesional T2 hyperintensity were noted. Lesion contact with either the meninges or ventricles was recorded. The presence of hemorrhage (determined by a combination of signal intensity on T1/T2-W and corresponding signal void on $\mathrm{T}^{*}$-gradient recall echo (GRE)), brain herniation (transtentorial or foramen magnum ${ }^{17}$ ), and mass effect (deviation of the falx cerebri from midline or compression of the lateral ventricle ${ }^{18}$ ) were recorded. The 
presence of cystic regions within the brain were recorded and defined as lesions being both strongly T1-hypointense and T2- hyperintense with sharply delineated margins on both sequences.

The lesion burden was estimated in 27 of the 30 dogs using a method described previously. ${ }^{19}$ In 3 dogs the image files were not compatible with the analysis program. Regions of interest (ROI) were drawn manually around lesions in every slice on the transverse $\mathrm{T} 2-\mathrm{W}$ to include perilesional edema in estimation of total lesion burden. Regions of interest were drawn around the entire brain in every slice on the transverse T2-W series. A DICOM-compatible software program (Image J 1.33u, Wayne Rashband, National Institutes of Health, USA) was used subsequently to calculate the number of voxels included in each ROI. For each animal the total number of lesion voxels was divided by the total number of brain voxels gave an estimation of lesion burden as a percentage of brain volume

Original brain tissue samples from each dog, obtained either from necropsy or biopsy, were sent to Texas A\&M University and evaluated by one pathologist (BP) to confirm tumor type. Representative hematoxylin and eosin-stained slides of each tumor were examined and graded according to the WHO animal grading system. ${ }^{20}$ An immunohistochemical stain for glial fibrillary acidic protein (GFAP), a marker of cells of astrocytic origin, was available in 7 cases. In general, the diagnosis of astrocytoma was made for those samples with round to ovoid to irregular nuclei with indistinct cell borders. Oligodendrogliomas were identified by round, hyperchromatic nuclei, prominent perinuclear halos, distinct cell borders, and branching capillaries. High grade tumors of both types had cellular pleomorphism, an increased mitotic rate, areas of 
necrosis, and tufts of prominent capillaries (microvascular proliferation) around the periphery of the neoplasm. Astrocytomas were classified as low, medium, or high grade, which are equivalent to grades II, III, and IV, respectively, in the WHO human classification. ${ }^{2}$ Oligodendrogliomas were classified as regular or anaplastic, which are equivalent to grades II and III in the human system. High grade tumors of both types had cellular pleomorphism, an increased mitotic rate, areas of necrosis, and tufts of prominent capillaries (microvascular proliferation) around the periphery of the neoplasm.

Data were summarized by calculating proportions and descriptive statistics and compared between tumor types. Quantitative variables, including lesion to:brain ratio, were dichotomized based on the median for estimation of proportions. Proportions were compared between tumor types and high versus low grade using Pearson chi-square or Fisher exact tests in available software (Epi Info, version 6.04, CDC, Atlanta, GA). Mann-Whitney $U$ tests were performed to compare quantitative variables between tumor types. Statistical analyses excluding the evaluation of categorical variables were performed in commercially available software (SPSS, version 17.0, SPSS Inc, Chicago, III.). All statistical results were interpreted at the $5 \%$ level of significance.

\section{Results}

There were no significant differences in clinical variables between the two tumor type groups: Proportion of female gender was not significant between tumor types $(P=$ 0.156). The median age (years) for dogs with astrocytomas was 9 (range 5-12) and oligodendrogliomas was 7 (range $5-13)(P=0.170)$. The median lesion:brain ratio for dogs with astrocytomas was 0.115 (range 0.042-0.327) and oligodendrogliomas was 
0.101 (range $0.020-0.172)(P=0.236)$. The median duration of signs (days) at the time of MR exam for dogs with astrocytomas was 16.5 (range 1-120) and oligodendrogliomas was 19 (range 1-270) $(P=0.813)$. Median survival (days) for dogs with astrocytomas was 78 (range 1-336) and for oligodendrogliomas was 73 (range 1487) $(P=0.822)$. Fourteen dogs survived to discharge (8 astrocytomas, 6 oligodendrogliomas). All dogs had died spontaneously or were euthanized by the time of the study.

All 16 oligodendrogliomas and 12 of 14 astrocytomas involved only the prosencephalon. One astrocytoma involved the cerebellum only, and one astrocytoma involved cerebellum and caudal brainstem. Frequency of occurrence among brain regions is reported in Table 1 . Only one dog in each tumor type had multiple brain lesions; the remainder had only singe lesions. Single tumors which involved more than one brain region appeared to extend continuously from one region to the next. Only three tumors crossed midline; all were astrocytomas ( 2 medium and 1 high grade).

MR imaging characteristics are summarized in Table 2. Precontrast tumor appearance is typified in Figure 1. Twenty-one of twenty-eight dogs given contrast medium had lesion enhancement graded as mild (3) astrocytomas, 6 oligodendrogliomas), moderate (4 astrocytomas, 4 oligodendrogliomas), or strong (3 astrocytomas, 1 oligodendroglioma) (Figure 3). Contrast enhancement was non-uniform in all dogs. Frequency of contrast enhancement was not significantly different between tumor types, but it was significantly more common in high grade tumors (Table 3 ). No other features were different between tumor grades (Table 3). 
Table 1. Proportion of Affected Brain Regions and Cerebral Lobes for 30 Dogs with Confirmed Astrocytoma or Oligodendroglioma.

\begin{tabular}{lccc}
\hline & Astrocytoma & Oligodendroglioma & \\
Location & Proportion & Proportion & P Value* \\
\hline Prosencephalon lesion & $12 / 14$ & $16 / 16$ & 0.209 \\
Frontal lobe & $10 / 14$ & $10 / 16$ & 0.709 \\
Olfactory lobe & $7 / 14$ & $6 / 16$ & 0.491 \\
Parietal lobe & $8 / 14$ & $6 / 16$ & 0.282 \\
Temporal lobe & $11 / 14$ & $10 / 16$ & 0.440 \\
Pyriform lobe & $7 / 14$ & $10 / 16$ & 0.491 \\
Hippocampus & $5 / 15$ & $7 / 17$ & 0.654 \\
Occipital lobe & $2 / 14$ & $2 / 16$ & 1.0 \\
Thalamus & $8 / 14$ & $4 / 16$ & 0.073 \\
Cerebellum lesion & $2 / 14$ & $0 / 16$ & 0.209 \\
Caudal brainstem lesion & $1 / 14$ & $0 / 16$ & 0.467 \\
\hline
\end{tabular}

*P value based on Pearson chi-square or Fisher exact tests. 
Table2 Proportion of Magnetic Resonance Imaging Findings in 30 Dogs with Astrocytoma or Oligodendroglioma.

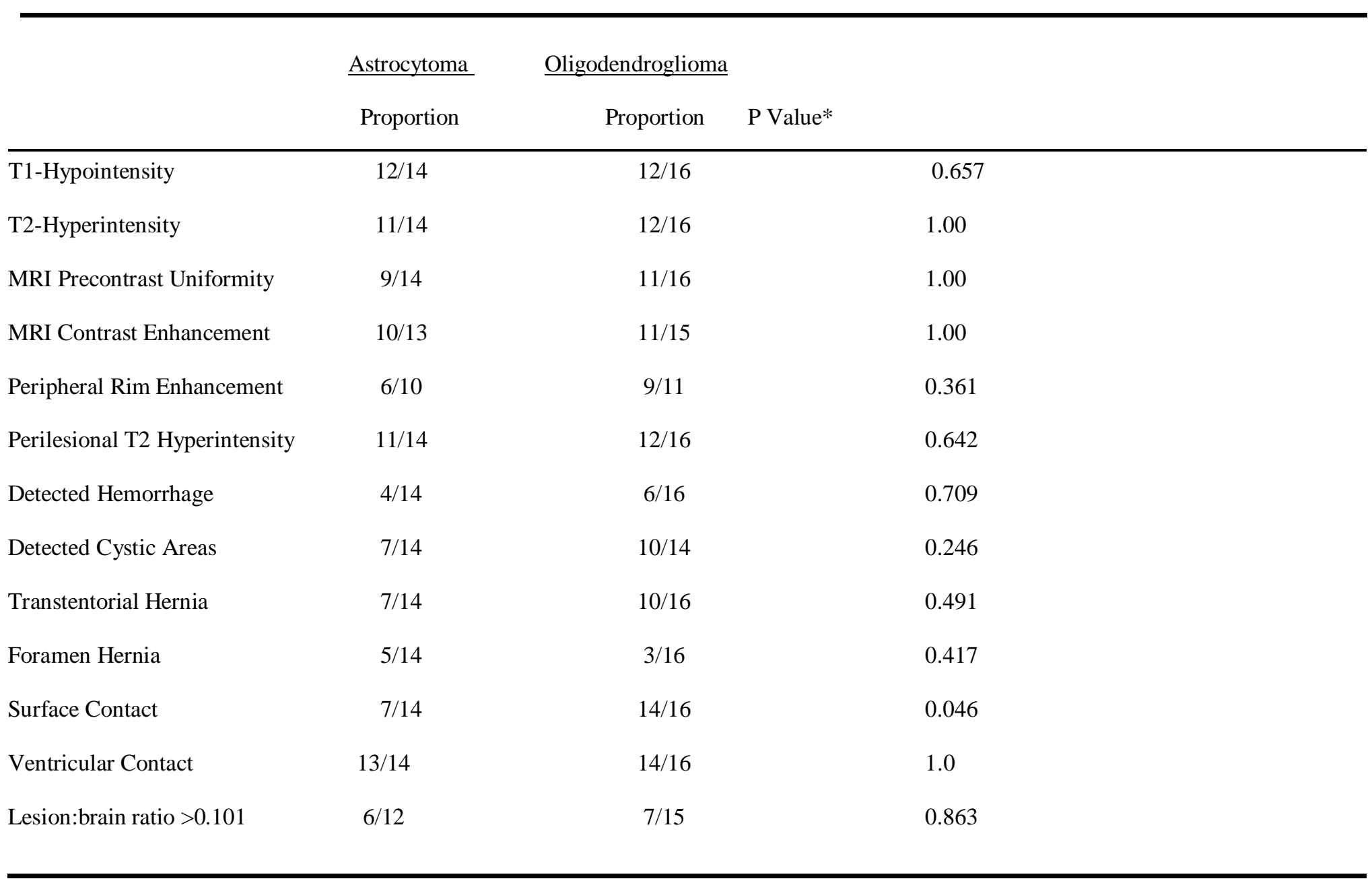

*P value based on Pearson chi-square or Fisher exact tests. 
Table 3. Comparison of magnetic resonance imaging findings for low grade (WHO Grade II) versus high grade (WHO Grades III\&IV) glioma in 30 dogs with astrocytoma or oligodendroglioma.

\begin{tabular}{|c|c|c|c|c|c|c|}
\hline \multirow[b]{2}{*}{ Variable } & \multicolumn{3}{|c|}{ Astrocytoma } & \multicolumn{2}{|c|}{ Oligodendroglioma } & \multirow[b]{2}{*}{ P Value* } \\
\hline & $\begin{array}{c}\text { WHO } \\
\text { Grade II } \\
\text { proportion }\end{array}$ & $\begin{array}{c}\text { WHO } \\
\text { Grade III } \\
\text { proportion }\end{array}$ & $\begin{array}{c}\text { WHO } \\
\text { Grade IV } \\
\text { proportion }\end{array}$ & $\begin{array}{c}\text { WHO } \\
\text { Grade II } \\
\text { proportion }\end{array}$ & $\begin{array}{c}\text { WHO } \\
\text { Grade III } \\
\text { proportion }\end{array}$ & \\
\hline T1-Hypointensity & $1 / 2$ & $5 / 6$ & $6 / 6$ & $2 / 3$ & $10 / 13$ & 0.254 \\
\hline T2-Hyperintensity & $2 / 2$ & $5 / 6$ & $4 / 6$ & $2 / 3$ & $11 / 13$ & 0.565 \\
\hline MRI Precontrast Uniformity & $0 / 2$ & $5 / 6$ & $4 / 6$ & $3 / 3$ & $8 / 13$ & 1.0 \\
\hline MRI Contrast Enhancement & $1 / 2$ & $4 / 5$ & $5 / 6$ & $0 / 3$ & $11 / 12$ & 0.008 \\
\hline Ring-like Enhancement & $0 / 1$ & $3 / 4$ & $3 / 5$ & $0 / 0$ & $9 / 11$ & 0.286 \\
\hline Perilesional T2 Hyperintensity & $1 / 2$ & $5 / 6$ & $5 / 6$ & $2 / 3$ & $12 / 13$ & 0.183 \\
\hline Detected Hemorrhage & $0 / 2$ & $1 / 6$ & $3 / 3$ & $2 / 3$ & $4 / 13$ & 1.0 \\
\hline Detected Cystic Areas & $1 / 2$ & $4 / 6$ & $2 / 6$ & $1 / 3$ & $11 / 13$ & 0.327 \\
\hline Transtentorial Hernia & $0 / 2$ & $4 / 6$ & $3 / 6$ & $2 / 3$ & $8 / 13$ & 0.628 \\
\hline Foramen Hernia & $0 / 2$ & $2 / 6$ & $3 / 6$ & $1 / 3$ & $2 / 13$ & 1.0 \\
\hline Meningial Contact & $2 / 2$ & $1 / 6$ & $4 / 6$ & $2 / 3$ & $12 / 13$ & 1.0 \\
\hline Ventricular Contact & $1 / 2$ & $6 / 6$ & $6 / 6$ & $2 / 3$ & $12 / 13$ & 0.064 \\
\hline Lesion:Brain Ratio >0.101 & $0 / 2$ & $2 / 5$ & $4 / 5$ & $2 / 3$ & $5 / 12$ & 1.0 \\
\hline
\end{tabular}

*P value based on Fisher exact tests comparing proportions within WHO Grade II to WHO Grades III and IV combined for both astrocytoma and oligodendroglioma. 
Astrocytomas were typically round or ovoid with defined margins (11/14) (Figure 1). Signal intensity was typically T1-hypointense and T2-hyperintense to grey matter, and most were uniform in intensity prior to contrast enhancement. All but two astrocytomas invaded or effaced both grey and white matter (Figure 1), while two appeared to involve only white matter. Half of the astrocytomas contacted the meninges (Figure 1), while all but one contacted the ventricles (Figure 2). Additionally seen features included perilesional T2-hyperintensity (11/14), cystic areas (7/14), and hemorrhage (4/14) Contrast enhancement was present in 10 of 13 cases and was ringlike in character in 6 of these cases (Figure 3).

Oligodendrogliomas were typically round or ovoid with defined margins $(13 / 16)$ (Figure 1). Signal intensity was typically T1-hypointense and T2-hyperintense to grey matter, and most were uniform in intensity prior to contrast enhancement. All but one oligodendroglioma invaded or effaced both grey and white matter (Figure 1), while one involved only grey matter. Thickening of the cortex was seen in only one tumor, a high grade oligodendroglioma (Figure 1C). Most oligodendrogliomas contacted the meninges (Figure 1) and the ventricles (Figure 2). One oligodendroglioma had broadly-based contact with the meninges. Additionally seen features included perilesional T2hyperintensity (12/16), cystic areas (12/16), and hemorrhage (6/16). Contrast enhancement was present in the 11 of 15 cases and was ring-like in 9 of these cases (Figure 3). 

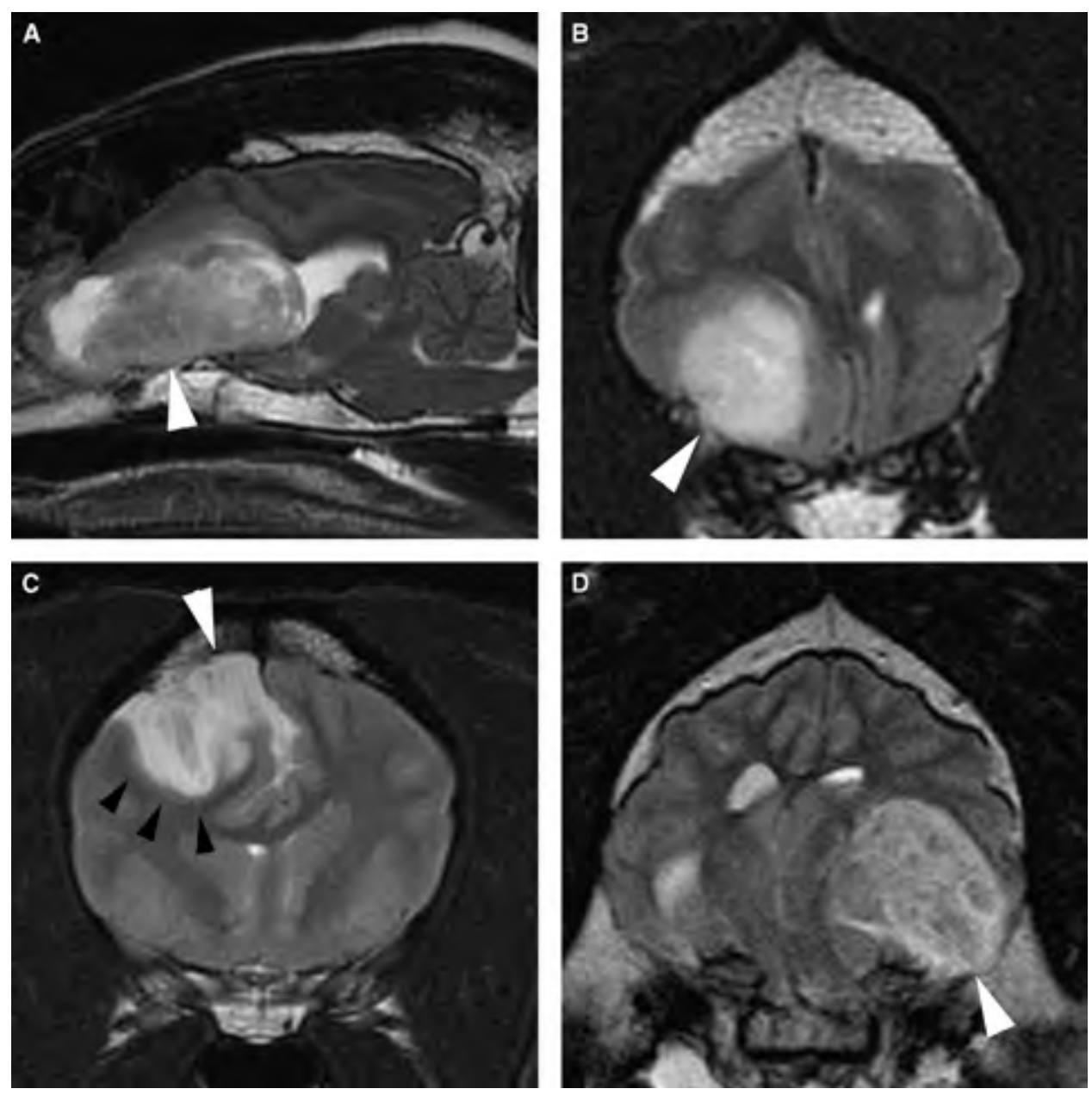

Figure 1. A) Sagittal T2-W in a dog with an astrocytoma. B) Transverse T2-W made at the level of the caudate nucleus in a dog with astrocytoma. C) Transverse T2W made at the level of the thalamus in a dog with oligodendroglioma. D) Transverse T2W made at the level of the hippocampus in a dog with oligodendroglioma. All of these lesions contact the meninges (white arrow heads) and are sharply defined. Examples A, $B$, and $D$ are ovoid and efface both grey and white matter. Example $C$ involves only the grey matter and there is thickening of the cortex (black arrow heads). 

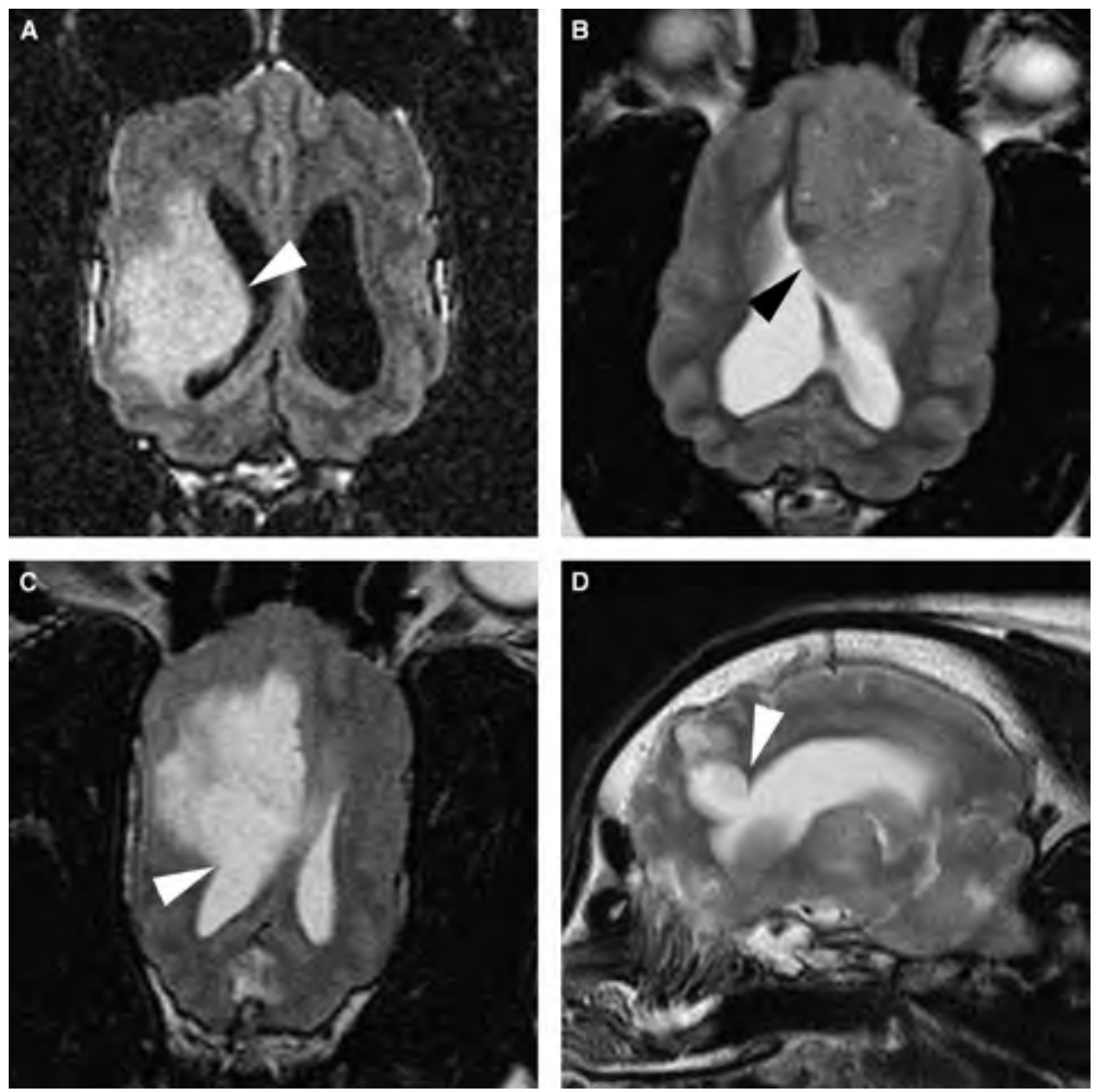

Figure 2. Dorsal T2-FLAIR (A) and T2-W (B) in 2 dogs with astrocytoma. Dorsal (C) and sagittal (D) T2-W in 2 dogs with oligodendroglioma. All lesions contact the lateral ventricles (arrow heads). Examples A, C, and D have typical T2-hyperintense signal, while B is atypically isointense to normal grey matter. Dramatic mass effect is present in $\mathrm{A}, \mathrm{B}$, and $\mathrm{C}$. 

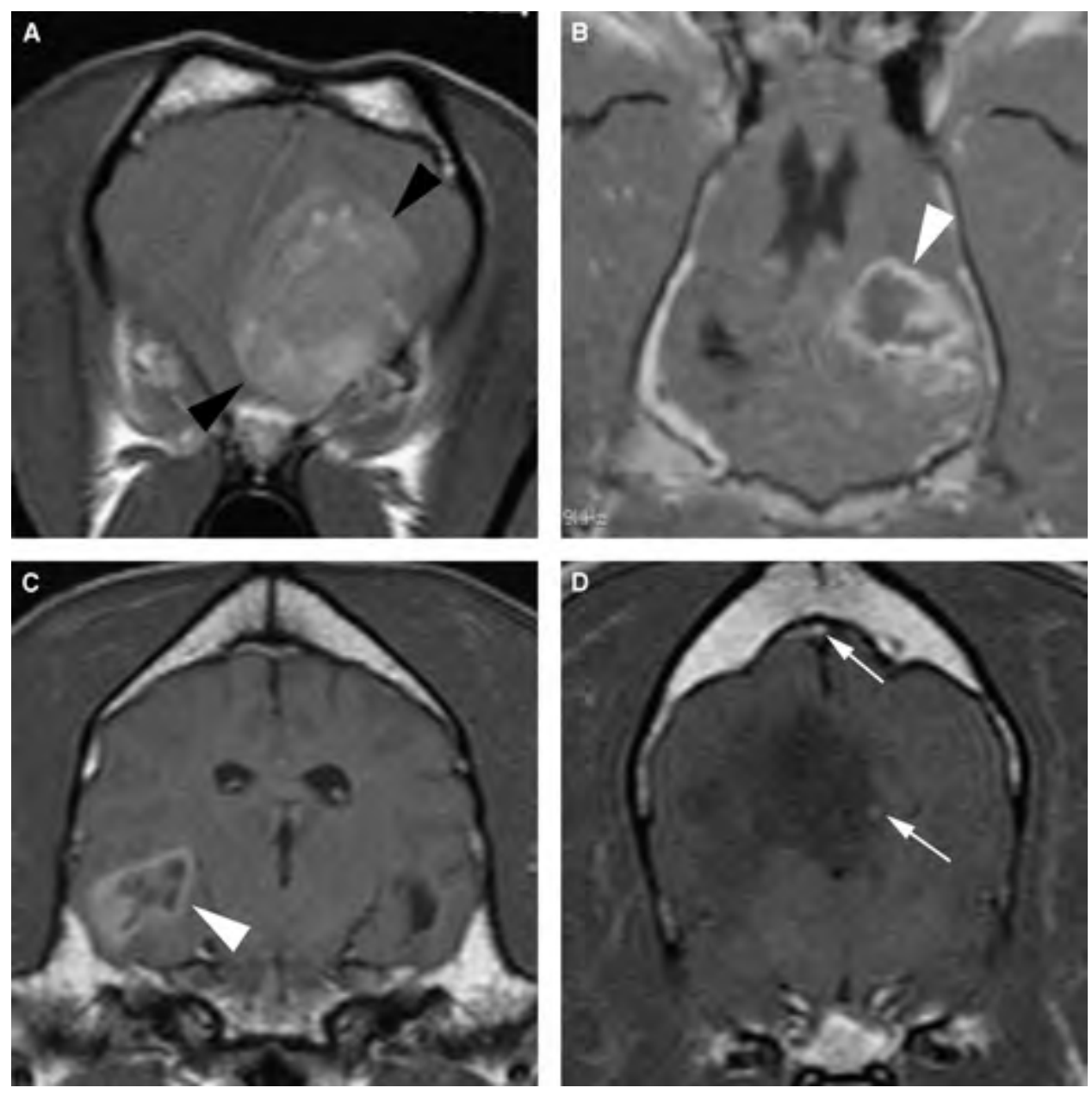

Figure 3. A) Transverse post-contrast T1-W at the level of the caudate nucleus in a dog with astrocytoma. B) Dorsal post-contrast T1-W in a dog with astrocytoma. C) Transverse post-contrast T1-W at the level of the hippocampus (C) and thalamus (D) in 2 dogs with oligodendroglioma. Lesion contrast enhancement is present in examples $\mathrm{A}$ (moderate and heterogeneous, black arrow heads), B and C (ring-like, white arrow heads). Example D has ill-defined, irregular margins and no contrast enhancement (note contrast medium in cerebral vessel and sinus, white arrows). 


\section{Discussion}

As antemortem histopathologic evaluation is uncommon in dogs with intracranial lesions, identifying the characteristic MR features of various diseases becomes important for estimating the diagnosis and formulating a treatment plan. In our evaluation of the MR features of astrocytoma vs. olidogendroglioma, both were intraaxial masses commonly involving grey and white matter of the prosencephalon. The lesions most commonly were T2-hyperintense, well marginated, ovoid, and enhanced following administration of contrast medium. Contact with the ventricular system was present in 27 of 30 tumors and lesion extension to the peripheral meninges was present in 21 of 30 tumors. Unfortunately, there were no features that allowed reliably differentiation of astrocytoma from oligodendroglioma, although there was an association between contrast enhancement and grade.

The material from the 30 dogs in this study was collected from four veterinary teaching hospitals in the continental United States. The breed distribution was consistent with previous reports, with Boxers and Boston Terriers being most frequently affected..$^{21}$ Dogs were generally middle age or older adults, typical of glioma patients. ${ }^{21}$ There was not a gender over-representation in dogs with glioma, which has been noted previously. ${ }^{21}$

All oligodendrogliomas and 12 of 14 astrocytomas involved only the prosencephalon, as expected. ${ }^{1}$ While all regions of the prosencephalon contained gliomas, a high proportion of lesions involved the piriform lobe and the adjacent temporal lobe. A tendency for canine glioma to be located in the piriform lobe has been suggested $^{22}$ but specific data have not been available previously. Additionally, there 
was glioma contact with ventricular surfaces in 27 of 30 dogs (Figure 2), a feature not reported previously in dogs. The propensity for gliomas to abut the ventricular system and involve the piriform lobe may underscore a potential mechanism of oncogenesis. Both the subventricular white matter and dentate gyrus, which closely approximates the piriform lobe, contain neural stem cells which may differentiate into gliomas in people and dogs. ${ }^{23-24}$

Tumor involvement of more than one brain region was rare $(2 / 30)$, as was identification of more than one brain lesion (2/30). Involvement of multiple brain regions has been reported in $79 / 168(47 \%)$ of canine brain tumors but types other than astrocytoma and oligodendroglioma were included. ${ }^{1}$ Only 3 of the 30 tumors presented here crossed midline, and all were astrocytomas. Though it would be reasonable to conclude that astrocytomas should be more extensive and more likely to involve a greater volume of brain, we found no difference in lesion burden between astrocytomas and oligodendrogliomas.

The most common appearance of each tumor type was that of an ovoid or spherical lesion with defined, regular margins. A minority of tumors in each group were amorphous with ill-defined, irregular margins. A mixture of defined and ill-defined lesions has been noted for astrocytomas and oligodendrogliomas. ${ }^{1,10-12}$ There were no MR features relating to signal intensity or uniformity that were significantly different between astrocytomas and oligodendrogliomas. Gliomas were generally T2hyperintense and T1-hypointense or isointense, which is non-specific. Ten of 30 tumors had non-uniform pre-contrast signal, which can be associated with hemorrhage, cyst formation, necrosis, altered cellular density, or mineralization. ${ }^{9}$ In people, a non-uniform 
lesion signal intensity is common in oligodendrogliomas and medium and high grade astrocytomas and is associated with the amount of tumor necrosis. ${ }^{9,25}$ Unlike in humans, a non-uniform lesion signal intensity did not add value in predicting tumor type or grade in dogs.

Cystic regions were identified in half of the astrocytomas and more than seventy percent of the oligodendrogliomas. This exceeds the frequency reported previously in canine gliomas. ${ }^{1,10,12-14,26}$ Cystic areas may develop for several reasons, including focal necrosis. ${ }^{12,27}$ MR findings suggestive of necrosis are low T1 signal, high T2 signal and lack of contrast enhancement. ${ }^{26,28}$ The use of T2-FLAIR sequences increases the sensitivity of tumor cyst detection over standard T1/T2-W sequences for lesions containing necrotic, proteinaceous or myxoid material. ${ }^{29}$ Tumor cysts may also contain debris, which settles to the dependant portion of the cyst during imaging. ${ }^{9}$ Cysts may be distinguished from a necrotic tumor focus by the presence of sharp margins and relatively higher T2 signal. ${ }^{9,28,30}$ However, while MR imaging is sensitive at detecting cysts and necrosis in dogs with gliomas, it was not sensitive enough to distinguish between them. ${ }^{12}$ Regardless of the cause, the identification of cystic regions in an intraaxial brain mass is a feature common to both astrocytomas and oligodendrogliomas in dogs. In contrast to humans, cysts did not occur more commonly in higher grade astrocytomas and was equally common to low grade varieties.

Hemorrhage was identified in 10 of 30 astrocytomas and oligodendrogliomas as a component of the larger mass. Hemorrhage associated with a brain tumor is often the result of necrosis and therefore typically has MR features which differ from benign hemorrhage.$^{9,31}$ Compared to a benign hematoma, tumor-associated hemorrhage often 
has heterogeneous signal from intermittent bleeding, associated non-hemorrhagic tissue that corresponds to the tumor, delayed hematoma evolution, absent hemosiderin rim and pronounced or persistent edema. ${ }^{31}$ Since sequential MR imaging is rare in canine patients, delay of hematoma evolution and prolonged edema can only be estimated using the onset of clinical signs as a temporal reference point. While MR recognition of mineralization has been described in gliomas, ${ }^{10}$ the appearance of brain tissue mineralization may be indistinguishable from hemorrhage without advanced MR techniques such as corrected gradient echo phase imaging and susceptibility weighted imaging. ${ }^{32-33}$ The attempt to identify mineralization on standard imaging sequences may be of little value in glioma differentiation.

Perilesional T2 hyperintensity was noted in greater than $75 \%$ of the astrocytomas and oligodendrogliomas. These results indicate a higher frequency of occurrence in oligodendrogliomas than thought previously. ${ }^{11}$ The term perilesional T2-hyperintensity was used instead of edema because in human gliomas, tumor margins often cannot be separated from edema and often exceed the margins established via imaging when evaluated with histopathology. ${ }^{9,34-35}$ Even with diffusion weighted MR imaging highgrade gliomas cannot be distinguished from surrounding edema. ${ }^{36}$ It has been suggested that the region of high T2 signal should be considered as tumor plus edema. ${ }^{9}$ Perilesional T2-hyperintensity was defined in our study as high T2 signal that appeared more extensive than the size of the mass based on the T1-hypointense region, or region of contrast enhancement. The MR detection of high perilesional T2 signal has been described for both gliomas and meningiomas and was not useful for distinguishing between these tumor types or for differentiating between neoplastic and non-neoplastic 
diseases in dogs. ${ }^{15}$ In human gliomas, the amount of perilesional T2-hyperintensity is one feature used to characterize glioma grade, ${ }^{9,25}$ This MR finding also is used as a prognostic indicator and has been linked to poorer survival in high-grade glioma. ${ }^{37-38}$ Contrary to our hypothesis and to the appearance in humans, we found no significant association between perilesional T2-hyperintensity and grade. The relationship of this finding to survival in dogs has yet to be established.

Lesion contrast enhancement was also present in approximately $75 \%$ of astrocytomas and oligodendrogliomas, and ranged from mild to strong. The incidence of ring-like enhancement was not different between the tumor types investigated here and is a non-specific finding common to many intracranial diseases. ${ }^{1,10-12,39-42}$ A linear relationship has been established between the degree of contrast enhancement and volume of peritumoral edema in human gliomas. ${ }^{43-44}$ The conclusion was that the same mechanism for breakdown of the blood brain barrier was the cause of both imaging findings. This perhaps explains the high rate of both findings in the tumors described herein. In our analysis, contrast enhancement was more common in higher grade gliomas and was the only feature which was significantly different between high and low grade tumors. This is similar to findings in diffuse astrocytomas in humans. ${ }^{25}$

The one feature which was significantly different between astrocytoma and oligodendroglioma was the appearance of tumor contact with the meninges. Oligodendrogliomas were significantly more likely to contact the brain surface than astrocytomas $(P=0.046)$. This may reflect a tendency for oligodendrogliomas to invade the brain periphery and meninges, as suggested previously. ${ }^{22}$ In humans, one distinguishing feature of adult oligodendroglioma is marked cortical thickening and 
infiltration. ${ }^{9}$ Thickening of the cortex was identified in one tumor in this study, a high grade oligodendroglioma. Histologically, all tumors which invaded the cortex and extended to the meninges appeared as thickened cortex. The smaller brain size and thinner cortex in dogs compared to people may be one explanation for the failure to detect this change. Alternatively the lack of apparent cortical thickening may reflect a difference between the biologic behavior of oligodendroglioma in dogs and people, as all but one oligodendroglioma involved both grey and white matter. It is possible that effacement of the grey-white distinction also obscures expansion of the cortex.

Although astrocytomas occurred more frequently in the diencephalon compared to oligodendrogliomas ( $57 \%$ versus $25 \%$, respectively), this difference was nonsignificant $(P=0.073)$, perhaps due to the sample size. In necropsy confirmed canine glioma, histologic involvement of the diencephalon was 8 times more likely in astrocytic tumors compared to oligodendroglial tumors. ${ }^{1}$

Survival was short for dogs with both glial tumor types, with a median less than 80 days. There was no significant difference in survival or the frequency of survival to discharge between dogs with astrocytoma vs. oligodendroglioma. Fifteen of the patients were euthanized shortly after imaging. Interestingly 10 of the 14 patients that survived to discharge were from the same institution. In fact 100 percent of the cases identified from that institution survived to discharge. These data suggest that clinician philosophy, means of patient follow-up, or research interests in addition to severity of neurologic deficits at the time of diagnosis ${ }^{3}$ influence survival time and will complicate the correlation of survival time with tumor type. 
Some limitations of our evaluation are as follows. The requirement for biopsy or necropsy confirmation limited the possibility of misclassification but might have causee selection bias if dogs without confirmation were different that those with confirmation. Imaging protocols were non-uniform and limited data were available on sequence variables but all studies were considered diagnostic. We made no attempt to correlate treatment to survival or the appearance of the tumor in the MR images because treatment data were not collected uniformly and were not available for all dogs. GFAP immunostaining was not available on all dogs. Differentiation between astrocytic and oligodendrocytic tumors can occasionally be difficult when based on H\&E morphology alone. Gliomas can be heterogeneous and have features of both astrocytoma and oligodendroglioma. The number of slides examined for each dog was small, and the slides may not have been representative of the entire neoplasm.

There is a need for non-invasive diagnostic criteria to distinguish gliomas from non-neoplastic brain diseases or less aggressive brain neoplasias. As brain biopsy is performed infrequently in dogs, MR imaging features are used commonly to make clinical diagnoses in intracranial disease. In one report, $70 \%$ of primary intracranial tumors in dogs could be grouped correctly as meningioma, glioma, choroid plexus papilloma, or ependymoma. ${ }^{12}$ However, $10 \%$ of the dogs had neoplastic disease which was misdiagnosed as a non-neoplastic process; all misdiagnosed dogs had glioma (3 of 15 total glioma cases). As a non-neoplastic disease comparison group was not included in the study presented here, the misdiagnosis of glial neoplasia might have been minimized artificially, and the accuracy of these MR features for differentiating glioma from non-neoplastic diseases remains unknown. 
Based on our findings, a solitary, well defined, contrast enhancing, intra-axial, ovoid, lesion of the prosencephalon with ventricular contact and mass effect are consistent with both astrocytoma or and oligodendroglioma. Ring-like contrast enhancement, perilesional T2-hyperintensity, cystic areas and hemorrhage also are common to both tumors. We did not identify an MR features which allows differentiation between these two tumor types, though oligodendrogliomas contact the peripheral meninges more frequently than do astrocytomas. Contrast enhancement was significantly associated with higher tumor grade.

\section{References}

1. Snyder JM, Shofer FS, Van Winkle TJ, et al. Canine intracranial primary neoplasia: 173 cases (1986-2003). J Vet Intern Med 2006;20:669-675.

2. Louis DN, Ohgaki H, Wiestler OD, et al. The 2007 WHO classification of tumours of the central nervous system. Acta Neuropathol 2007;114:97-109.

3. Heidner GL, Kornegay JN, Page RL, et al. Analysis of survival in a retrospective study of 86 dogs with brain tumors. J Vet Intern Med 1991;5:219-226.

4. Julia-Sape M, Acosta D, Majos $C$, et al. Comparison between neuroimaging classifications and histopathological diagnoses using an international multicenter brain tumor magnetic resonance imaging database. J Neurosurg 2006;105:6-14.

5. Shaw EG, Scheithauer BW, O'Fallon JR, et al. Oligodendrogliomas: the Mayo Clinic experience. J Neurosurg 1992;76:428-434.

6. Ohgaki $\mathrm{H}$, Kleihues P. Population-based studies on incidence, survival rates, and genetic alterations in astrocytic and oligodendroglial gliomas. J Neuropathol Exp Neurol 2005;64:479-489.

7. De Stefani A, Sparkes A, Garosi LS, et al. Clinical signs, magnetic resonance imaging findings and survival in dogs with intracranial meningiomas and glial cell tumors. J Vet Intern Med 2009;23:737.

8. Rossmeisl J, Robertson $\mathrm{J}$, Jones $\mathrm{J}$, et al. Effects of palliative pharmacotherapy on survival and quality of life in 50 dogs with primary intracranial neoplasms. $J$ Vet Intern Med 2009;23:708.

9. Jayaraman MV, Boxerman JL. Adult Brain Tumors In: Atlas SW, ed. Magnetic Resonance Imaging of the Brain and Spine. 4th ed. Philadelphia: Lippincott Williams \& Wilkins, 2009;445-590.

10. Kraft SL, Gavin PR, DeHaan C, et al. Retrospective review of 50 canine intracranial tumors evaluated by magnetic resonance imaging. $J$ Vet Intern Med 1997;11:218-225.

11. Kraft SL, Gavin PR. Intracranial neoplasia. Clin Tech Small Anim Pract 1999;14:112-123. 
12. Rodenas S, Pumarola M, Gaitero L, et al. Magnetic resonance imaging findings in 40 dogs with histologically confirmed intracranial tumours. Vet $J 2009$.

13. Thomas WB, Wheeler SJ, Kramer R, et al. Magnetic Resonance Imaging Features of Primary Brain Tumors in Dogs. Veterinary Radiology and Ultrasound 1996;37:20-27.

14. Kraft SL, Gavin PR, Leathers CW, et al. Diffuse cerebral and leptomeningeal astrocytoma in dogs: MR features. J Comput Assist Tomogr 1990;14:555-560.

15. Cherubini GB, Mantis P, Martinez TA, et al. Utility of magnetic resonance imaging for distinguishing neoplastic from non-neoplastic brain lesions in dogs and cats. Vet Radiol Ultrasound 2005;46:384-387.

16. Leigh EJ, Mackillop E, Robertson ID, et al. Clinical anatomy of the canine brain using magnetic resonance imaging. Vet Radiol Ultrasound 2008;49:113-121.

17. Walmsley GL, Herrtage ME, Dennis R, et al. The relationship between clinical signs and brain herniation associated with rostrotentorial mass lesions in the dog. Vet $J$ 2006;172:258-264.

18. Kii S, Uzuka $\mathrm{Y}$, Taura $\mathrm{Y}$, et al. Magnetic resonance imaging of the lateral ventricles in Beagle-type dogs. veterinary Radiology and Ultrasound 1997;38:430-433.

19. Young BD, Levine JM, Fosgate GT, et al. Magnetic resonance imaging characteristics of necrotizing meningoencephalitis in Pug dogs. $J$ Vet Intern Med 2009;23:527-535.

20. Koestner A BT, Fatzer R, Schulman FY, Summers BA, Van Winkle TJ. Histologic Classification of Tumors of the Nervous System of Domestic Animals In: Pathology AFlo, ed. Washington D.C, 1999.

21. Hayes HM, Priester WA, Jr., Pendergrass TW. Occurrence of nervous-tissue tumors in cattle, horses, cats and dogs. Int $J$ Cancer 1975;15:39-47.

22. Fankhauser R, Luginbuhl H, McGrath JT. Tumours of the nervous system. Bull World Health Organ 1974;50:53-69.

23. Stoica $\mathrm{G}$, Lungu $\mathrm{G}$, Martini-Stoica $\mathrm{H}$, et al. Identification of cancer stem cells in dog glioblastoma. Vet Pathol 2009;46:391-406.

24. Sanai N, Alvarez-Buylla A, Berger MS. Neural stem cells and the origin of gliomas. N Engl J Med 2005;353:811-822.

25. Tervonen O, Forbes G, Scheithauer BW, et al. Diffuse "fibrillary" astrocytomas: correlation of MRI features with histopathologic parameters and tumor grade. Neuroradiology 1992;34:173-178.

26. Lipsitz D, Higgins RJ, Kortz GD, et al. Glioblastoma multiforme: clinical findings, magnetic resonance imaging, and pathology in five dogs. Vet Pathol 2003;40:659-669.

27. Bagley RS, Kornegay JN, Lane SB, et al. Cystic meningiomas in 2 dogs. $J$ Vet Intern Med 1996;10:72-75.

28. Pierallini A, Bonamini M, Pantano $\mathrm{P}$, et al. Radiological assessment of necrosis in glioblastoma: variability and prognostic value. Neuroradiology 1998;40:150-153.

29. Aprile I, laiza F, Lavaroni A, et al. Analysis of cystic intracranial lesions performed with fluid-attenuated inversion recovery MR imaging. AJNR Am J Neuroradiol 1999;20:1259-1267.

30. Kjos BO, Brant-Zawadzki M, Kucharczyk W, et al. Cystic intracranial lesions: magnetic resonance imaging. Radiology 1985;155:363-369. 
31. Atlas SW, Grossman RI, Gomori JM, et al. Hemorrhagic intracranial malignant neoplasms: spin-echo MR imaging. Radiology 1987;164:71-77.

32. Thomas B, Somasundaram S, Thamburaj K, et al. Clinical applications of susceptibility weighted MR imaging of the brain - a pictorial review. Neuroradiology 2008;50:105-116.

33. Gupta RK, Rao SB, Jain R, et al. Differentiation of calcification from chronic hemorrhage with corrected gradient echo phase imaging. J Comput Assist Tomogr $2001 ; 25: 698-704$.

34. Earnest Ft, Kelly PJ, Scheithauer BW, et al. Cerebral astrocytomas: histopathologic correlation of MR and CT contrast enhancement with stereotactic biopsy. Radiology 1988;166:823-827.

35. Kelly PJ, Daumas-Duport C, Kispert DB, et al. Imaging-based stereotaxic serial biopsies in untreated intracranial glial neoplasms. J Neurosurg 1987;66:865-874.

36. Castillo M, Smith JK, Kwock L, et al. Apparent diffusion coefficients in the evaluation of high-grade cerebral gliomas. AJNR Am J Neuroradiol 2001;22:60-64.

37. Pope WB, Sayre J, Perlina A, et al. MR imaging correlates of survival in patients with high-grade gliomas. AJNR Am J Neuroradiol 2005;26:2466-2474.

38. Carlson MR, Pope WB, Horvath $S$, et al. Relationship between survival and edema in malignant gliomas: role of vascular endothelial growth factor and neuronal pentraxin 2. Clin Cancer Res 2007;13:2592-2598.

39. Cherubini GB, Platt SR, Anderson TJ, et al. Characteristics of magnetic resonance images of granulomatous meningoencephalomyelitis in 11 dogs. Vet Rec 2006;159:110-115.

40. von Praun F, Matiasek K, Grevel V, et al. Magnetic resonance imaging and pathologic findings associated with necrotizing encephalitis in two Yorkshire terriers. Vet Radiol Ultrasound 2006;47:260-264.

41. Kastrup O, Wanke I, Maschke M. Neuroimaging of infections. NeuroRx 2005;2:324-332.

42. Wolf $\mathrm{M}$, Pedoria $\mathrm{V}$, Higgins RJ, et al. Intracranial ring enhancing lesions in dogs: a correlative CT scanning and neuropathologic study. Vet Radiol Ultrasound 1995;36:16-20.

43. Holodny AI, Nusbaum AO, Festa $S$, et al. Correlation between the degree of contrast enhancement and the volume of peritumoral edema in meningiomas and malignant gliomas. Neuroradiology 1999;41:820-825.

44. Pronin IN, Holodny AI, Petraikin AV. MRI of high-grade glial tumors: correlation between the degree of contrast enhancement and the volume of surrounding edema. Neuroradiology 1997;39:348-350. 